\title{
Validation of Chinese version of the 4-item Trust in Nurses Scale in patients with cancer
}

\author{
This article was published in the following Dove Press journal: \\ Patient Preference and Adherence \\ 2 November 2017 \\ Number of times this article has been viewed
}

\section{Ling Zhao ${ }^{1,2}$ \\ Rong Wang ${ }^{3}$ \\ Shan Liu ${ }^{4}$ \\ Jin $\operatorname{Yan}^{5}$}

'Department of Clinical Psychological Nursing, Xiangya Nursing School, Central South University, Changsha, ${ }^{2}$ Department of International and Humanistic Nursing, School of Nursing, University of South China, ${ }^{3}$ Department of Oncology, ${ }^{4}$ Department of Hematology, The First Affiliated Hospital of University of South China, Hengyang, ${ }^{5}$ Department of Nursing, The Third Xiangya

Hospital, Changsha, China
Correspondence: Jin Yan

Department of Nursing, The Third Xiangya Hospital, 138 Tongzipo Road,

Changsha 410013, China

Tel +86073 I 886I 8626

Email yanjin0163@।63.com
Objective: This study was designed to examine the internal consistency, test-retest reliability, construct, and concurrent validity of the Trust in Nurses Scale (TNS) in hospitalized patients with cancer in China.

Methods: Between October and December 2016, the Chinese version of TNS and Nurse-Patient Trust Scale were applied to assess 190 patients with cancer in a general hospital. A subsample of 70 patients completed the TNS again 1 week later.

Results: The Chinese version of the TNS had good internal consistency ( $\alpha=0.817)$, fair testretest reliability $(r=0.866)$, and confirmatory factor analysis demonstrated good fit for a fouritem version of the TNS.

Conclusion: The Chinese TNS exhibited sufficient validity and reliability in hospitalized patients with cancer.

Keywords: trust, measurement, nurse-patient relationship, reliability, validity, confirmatory factor analysis

\section{Background}

Cancer is a major cause of morbidity and mortality, according to the World Health Organization, with $\sim 14$ million new cases and 8 million cancer-related deaths in 2012 . Most of the newly reported cancers were in the developing world, and new cancer cases ranked first in China in 2012. ${ }^{1}$ The National Central Cancer Registry of China predicted that 4,292,000 new cancer cases and 2,814,000 cancer deaths would occur in China in $2015 .^{2}$ Given the complexity and duration of cancer care episodes, it is important to develop a good relationship with caregivers, which includes trust. ${ }^{3}$ Trust is more required while in life threatening situations. ${ }^{4}$ Therefore, cancer patients required more trust during their cancer trajectory.

Bell and Duffy ${ }^{5}$ defined trust as "the optimistic acceptance of a vulnerable situation, following careful assessment, in which the truster believes that the trustee has his best interests as paramount". Nurses are the closest health care providers to patients. The nurse-patient relationship is central to the discipline of nursing, and it is responsible for the association of positive health outcomes. ${ }^{6}$ A trust in nurse-patient relationship is demonstrated where patient is confident in and relies on the nurses' care. ${ }^{7}$ Trust plays an essential role in nurse-patient relationship because without trust, it is not possible to effectively meet the needs of patients and to improve their satisfaction with nursing care. ${ }^{8}$

From the patients' perspective, trust has positive associations with patient satisfaction, receipt of care in nurse-managed center, the well-being of a birthing woman and her child, patient competence of illness management, and adjustment to illness for 
dying patients. ${ }^{9-12}$ Good trust has been shown to be associated with better job satisfaction, good organizational citizenship behaviors, and organizational commitment for nurses. ${ }^{13,14}$ Therefore, trust affects the quality of patient care, which is associated with an improvement of patient outcomes during nursing care episodes.

Trust is at the heart of nurse-patient relationships; measurement of trust with a reliable and valid instrument is important. Since 1990s, the measurement of nursepatient trust relationship has attracted attention in China. Nurse-patient Trust Scale (NPTS), which was developed by a Japanese scholar, has been translated into Chinese and applied in clinical practice. ${ }^{15}$ The scale's reliability and validity have been tested in 2010; research indicated that it needed to be further modified according to the situation of China. ${ }^{16}$ However, there is scarcely research on revision of the NPTS until now. Due to the large number of items and ambiguous wording, respondents may experience difficulty in completing the scale.

The Trust in Nurses Scale (TNS) was developed to measure cancer patients' trust in nurses by addressing nurses' activities and patient feelings. The original version of TNS consisted of one global and five items and demonstrated excellent item-convergent validity $(0.50 \leq r \leq 0.73)$ and internal consistency $(\alpha=0.81)$ in a sample of 66 ambulatory patients with cancer. ${ }^{17}$ The English TNS scale has two versions, such as 5-item and 4-item TNS, both of which have acceptable reliability and validity (Cronbach's $\alpha$ coefficient for TNS-5 is 0.77 and Cronbach's $\alpha$ coefficient for TNS-4 is 0.82$).{ }^{18}$ The English version of TNS-4 has been translated into Finnish, Swedish, and Greece, and its psychometric properties have been verified. ${ }^{19}$ To date, no studies have investigated the application of this scale in China. Moreover, the test-retest reliability of the scale has not yet been tested. This study aimed to assess the reliability and validity of Chinese version of TNS-4 in hospitalized patients in an oncology setting.

\section{Sample and setting}

Data were collected from patients hospitalized for cancer at the First Affiliated Hospital of University of South China, Hengyang City, Hunan Province, China, between October and December 2016.

Potential participants were approached by a nurse, who inquired if they were interested in learning about the study from a research team member. Eligible patients were invited to participate if they 1) were aged 18 years or older, 2) had a confirmed histopathological cancer diagnosis, 3) had received hospital care as in-patients for at least $48 \mathrm{~h}$, and 4) were capable of answering the questionnaire independently. Patients with cognitive impairments or literacy problems or not aware of their cancer diagnosis were excluded. Assenting patients were approached by a researcher. A cover letter and consent form explaining the purpose and voluntary nature of the study accompanied each questionnaire. At the end of survey, patients were asked whether they were willing to complete the TNS a second time 1 week later if they were in hospital.

A total of 190 participants comprised the sample for constructing validity testing. The sample size was sufficient to estimate the eight parameters (four path coefficients and four variances for the four items analyzed) using the $\mathrm{N}$ : q rule of 10-20 subjects per parameter. ${ }^{20}$

This study was approved by the First Affiliated Hospital of University of South China Ethics Committee, and the written informed consent was obtained before the survey.

\section{Measures TNS}

In the study, the 4-item TNS was adopted and the English version of the TNS was translated into the Chinese version after obtaining consent from the author. A forward-back translation protocol was adopted. ${ }^{21}$ First, an official translator translated the scale from English to Chinese. The first translation was checked by investigators. Second, the backtranslation was produced by other translators. The English and Chinese versions were reconciled by both Chinese- and English-speaking investigators. A pilot test was conducted to confirm the readability and feasibility of the scale in the actual survey by recruiting 10 eligible patients. Chinese version of TNS consists of four items, it has a 5-point response scale from 1 (never) to 5 (always). A high score represents high trust in nurses.

\section{NPTS}

The NPTS was a 41-item self-report scale designed to measure patients' levels of trust in nurses and was divided into the following five subscales: assurance to knowledge and technique (7 items), consistency (11 items), respect (11 items), reassurance ( 7 items), and trust to future (5 items). All questions are rated on 4-point Likert-type scale from "disagree" to "completely agree". Scores are summed to range from 41 to 164, with higher scores indicating patients' better trust in nurses. ${ }^{15}$ The Cronbach's $\alpha$ coefficient of Chinese version was $0.89 .{ }^{16}$

\section{Statistical analysis}

Descriptive and basic psychometric analyses were performed using SPSS 23.0, whereas structural validity was examined 
using confirmatory factor analysis (CFA) with AMOS 21.0. Prior to CFA, an exploratory factor analysis (EFA) was conducted using the Kaiser-Meyer-Olkin (KMO) measure of sampling adequacy to assess for sufficient sample size and Bartlett's test of sphericity to test for the homogeneity of variances. The number of missing values for the TNS was very low; only 2 missing values for item 2 , missing values were imputed with the median value. Loadings of the items on the latent factor were estimated using a maximumlikelihood analysis with data from the 190 patients. The goodness-of-fit index (GFI), Tucker-Lewis index (TLI), root mean square residual (RMR), and the root mean square error of approximation (RMSEA) were used to examine the model fit. GFI and TLI values $>0.9$, RMR values $>0.05$, and RMSEA values $<0.08$ were considered indicative of good model fit. ${ }^{22,23}$

The reliability of the Chinese version of the TNS was assessed using Cronbach's $\alpha$ coefficient, where a value of $\geq 0.70$ was considered acceptable internal consistency. ${ }^{24}$ The corrected item-to-total correlations and split-half reliability coefficient were also used to evaluate reliability, while a value of $>0.3$ and $P<0.05$ were adopted as criteria. ${ }^{25,26}$ Test-retest reliability was assessed by computing the intraclass correlation coefficient (ICC) with $95 \%$ confidence intervals, and the ICC $\geq 0.70$ was considered acceptable external consistency. ${ }^{27}$ Bland-Altman plot was used in analyzing the agreement between test and retest scores of TNS. ${ }^{28}$ Concurrent validity was evaluated by examining Pearson's correlations with NPTS. Pearson correlations $<0.3$ were considered weak, correlations between 0.3 and 0.5 were considered moderate, and correlations $>0.5$ were considered strong. ${ }^{29}$

\section{Results}

\section{Patients}

Of the 215 participants recruited, 190 (90.5\%) patients returned a completed survey and 70 patients completed the TNS a second time. Of the study participants, 97 participants were males and the mean age was 46 years. Patients had various types of cancer, the four most frequently occurring being lung, hematological, colorectal, and breast. Table 1 displays the demographic and clinical characteristics of participants.

\section{Distribution}

Total scores on the TNS showed a near-normal distribution (Figure 1). The overall score of TNS for the patients was a little higher than the medium. Although the scale was slightly skewed toward higher scores, absolute skewness $(-0.522)$
Table I Sample characteristics (N=190)

\begin{tabular}{ll}
\hline Characteristics & Mean \pm SD or number (\%) \\
\hline Age, years & $46 \pm 13.2$ \\
Sex, male & $97(51.1)$ \\
Ethnicity, Han nationality & $186(97.9)$ \\
Marital status & $152(80.0)$ \\
$\quad$ Married & $20(10.5)$ \\
$\quad$ Not married & $18(9.5)$ \\
$\quad$ Widowed/divorced & \\
Education & \\
$\quad$ Low & $25(13.1)$ \\
$\quad$ Medium & $98(51.6)$ \\
$\quad$ High & $67(35.3)$ \\
Type of admission & $154(81.1)$ \\
$\quad$ Planned & $36(18.9)$ \\
Emergency admission & $172(90.5)$ \\
Previous experiences of hospital & $18(9.5)$ \\
$\quad$ Yes & $15.4 \pm 3.4$ \\
$\quad$ No & $127.4 \pm 17.1$ \\
TNS (range 4-20) &
\end{tabular}

Notes: ${ }^{\text {L }}$ Low $=$ none, primary school; medium = middle-level vocational training, higher level secondary general education; high = higher level vocational training, academic education.

Abbreviations: NPTS, Nurse-Patient Trust Scale; TNS, Trust in Nurses Scale.

and kurtosis $(-0.567)$ were well $<1$. None of patients scored 4 points, and 24 patients (12.6\%) obtained a score of 20 points. In this study, since no more than $15 \%$ of the patients scored the worst or best possible score on the TNS, respectively, floor and ceiling effects were acceptable. ${ }^{30}$

\section{Internal consistency and test-retest reliability}

For internal consistency, the Cronbach's $\alpha$ coefficient was 0.765 ; for split-half reliability, the Spearman-Brown coefficient was $0.745(P<0.05)$ and Guttman split-half coefficient

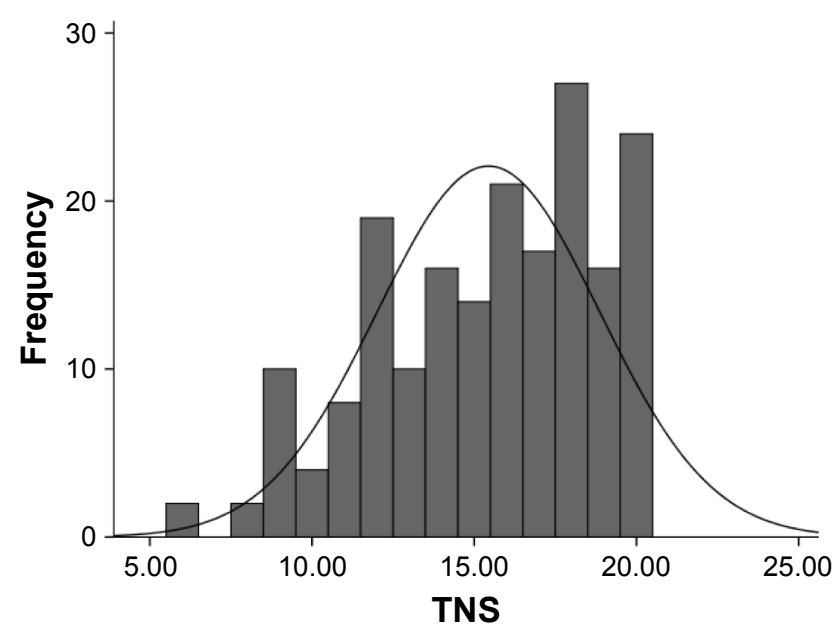

Figure I Distribution of total TNS scores. Abbreviation: TNS, Trust in Nurses Scale. 


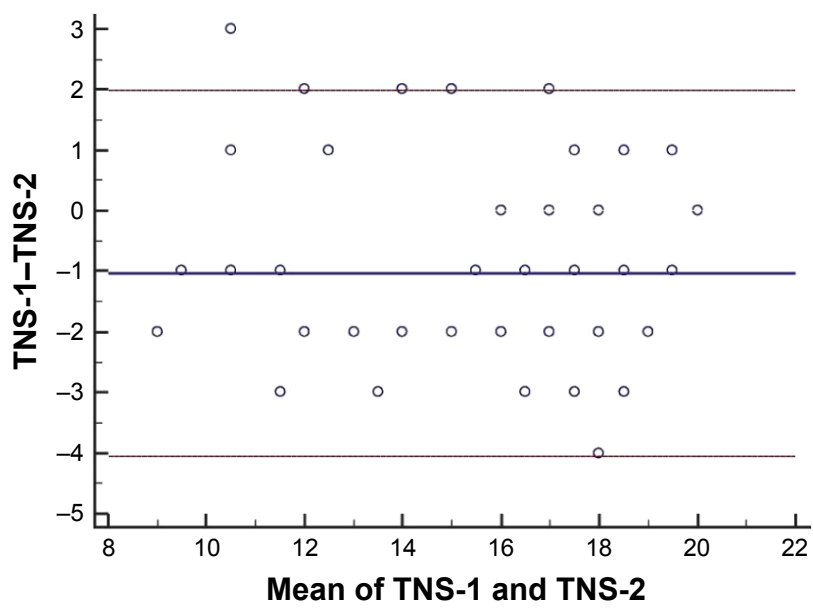

Figure 2 Individual agreement between test and retest scores of the TNS. Notes: The horizontal solid line represents the mean difference between both scores. The dashed line represents the $95 \%$ limits of agreement (mean difference 1.96 \pm SD of the difference). TNS-I means the first test of TNS; TNS-2 means the re-test of TNS. Abbreviation: TNS, Trust in Nurses Scale.

was $0.743(P<0.05)$. The item-total correlations of the TNS ranged from 0.641 to 0.728 . With an ICC of $0.87(95 \% \mathrm{CI}$ : 0.79-0.91), test-retest reliability (0.866) demonstrated good consistence between two different times. Bland-Altman analysis (Figure 2) demonstrated that the limits of agreement between the two time points ranged from -4.05 to 1.99 , only $1.4 \%(1 / 70)$ of point beyond the $95 \% \mathrm{CI}$, the TNS showed good agreement over time. Although the reproducibility was tested with a subsample, the baseline TNS scores of patients who attended the re-test assessment were higher

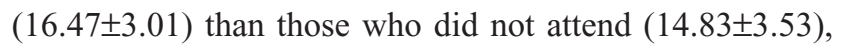
there was no significant difference between two groups $(t=3.253, P=0.08)$.

\section{Structural validity and concurrent validity}

For EFA of construct validity, the KMO value was 0.762 , Bartlett's test was statistically significant $\left(\chi^{2}=183.905\right.$, $P<0.001$ ), where factor analysis was applicable. The principal component analysis supported the one component structure of the TNS in China. Communalities were above the recommended value of $>0.2 .{ }^{31}$ One common factor with eigenvalue (2.356) was extracted using principal component analysis, and the cumulative contribution rate was $60.91 \%$ (Table 2 and Figure 3). CFA showed good fit indices for 1 -factor model of the TNS $\left(\chi^{2}=3.451\right.$, df $=2$, GFI $=0.991$, $\mathrm{CFI}=0.992$, TLI $=0.976$, RMR $=0.028$, RMSEA $=0.062$ ). Standardized factor loadings ranged between 0.62 for item 4 and 0.76 for item 2 (Figure 4). The 4-item TNS had good concurrent validity with the NPTS $(r=0.766)$.
Table 2 Factor analysis of the Chinese version of TNS

\begin{tabular}{lll}
\hline Item no & Element matrix & Communality \\
\hline $\mathrm{I}$ & 0.738 & 0.544 \\
2 & 0.812 & 0.659 \\
3 & 0.774 & 0.599 \\
4 & 0.744 & 0.554 \\
Percentage of explained variance & 60.91 & \\
Component suggested & $\mathrm{I}$ & \\
KMO & 0.762 & \\
Bartlett's test, chi-square $(P$-value $)$ & $\mathrm{I} 83.905(<0.00 \mathrm{I})$ & \\
\hline
\end{tabular}

Abbreviations: KMO, Kaiser-Meyer-Olkin; TNS, Trust in Nurses Scale.

\section{Discussion}

In this study, the patients' trust in nurses was considered above average, which is a little lower than previous studies. ${ }^{3,19}$ The reason for this may be the cultural background and political situations. In China, government funding to the public hospitals is $\sim 5 \%-10 \%$; in order to survive the competition, public hospitals have to keep operating and obtain economic profits by using various "income generation"; this may reduce the public trust. ${ }^{32}$ Public trust will impact patients' trust to health care providers. In addition, shortage of personnel also contributed to it. According to the Health and Family Planning statistic bulletin, practicing registered nurses per population was 2.36 in China, lower than the previous studies. ${ }^{33}$ Trust develops in relationships, and it takes time to build trust. ${ }^{34}$ Busy work and lack of staff reduced nurses' opportunities of communication, and less time spent with patients had an impact on the study results.

The main purpose of this study was to introduce the TNS into China and to evaluate its reliability and validity in a small

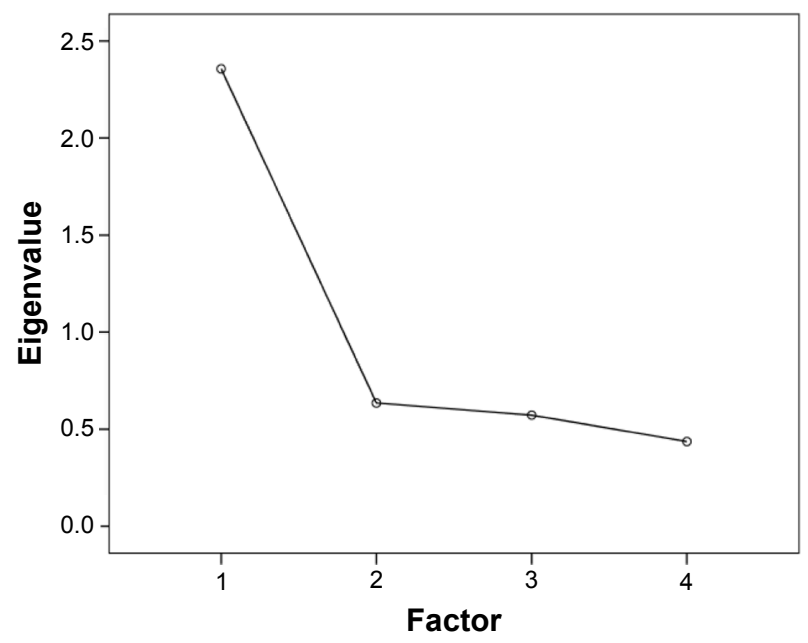

Figure 3 Screen plot.

Note: One factor with eigenvalue $\geq I$ was extracted using principal component analysis. 


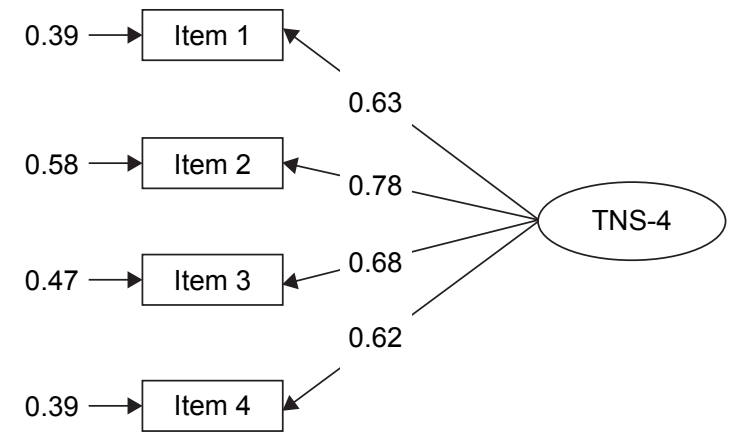

Figure 4 Standardized factor loadings and residuals for the items of the TNS-4. Abbreviation: TNS, Trust in Nurses Scale.

sample of hospitalized patients with cancer. Our results indicate that the TNS-4 has good reliability in patients hospitalized for cancer; the Cronbach's $\alpha$ coefficient of the Chinese version of TNS was similar to the English (0.77 in 2010 and 0.81 in 2005), Finnish (0.78), and Greece (0.84) versions. ${ }^{17-19}$ Cronbach's $\alpha$ coefficients are sensitive to the number of items, and thus, it may be an inappropriate index for a very short test. ${ }^{35}$ Item-total correlations or split-half reliability can provide a useful index for a very short scale. ${ }^{36}$ In our study, the item-total correlations and split-half reliability were conducted to confirm the internal consistency of the TNS. The findings of the item-total correlations (0.641-0.728) and split-half reliability $(0.743)$ met the quality criteria.

Test-retest reliability was used to ascertain the degree of change in the scores over time, which provided further evidence of the reliability of TNS. The ICC for absolute agreement was 0.87 , which is higher than the quality criteria (0.7). ${ }^{27}$ The Bland-Altman analysis further illustrated the good test-retest reliability. One week between the repeated administrations was used in our study with the consideration of the cancer patients' hospital stays. Most of the patients (77.1\%) who completed the second survey are from hematology department, and they may have previous hospital experiences, which may have resulted in high level trust in nurses. This may result in bias. Moreover, the high testretest reliability may result from shorter time interval. Future studies should examine test-retest reliability over a longer time interval and in different groups of people.

Factor analysis results confirmed that the TNS-4 measured a unidimensional construct and indicated that the items of the TNS demonstrated acceptable goodness-of-fit to a 1 -factor model. The previous studies confirmed good fit for a unidimensional model of the TNS-4 using Mplus and WINSTEPS Rasch analysis software. ${ }^{18,19}$ To the best of our knowledge, this is the first study that examines the construct and concurrent validity of the Chinese version of TNS-4. The factor loading value is the correlation between a variable and a factor, and scores $>0.4$ are acceptable. ${ }^{37}$ In our study, the factor loadings (range $0.62-0.78$ ) were above the cutoff, which showed that variables were closely associated with the TNS. There is no universally agreed gold standard for the percentage of explained variance, but our result $(60.91 \%)$ was parallel with the values of previous studies. ${ }^{18,19}$

There is no gold standard scale to measure trust relationship between nurse and patient, but at present, the NPTS is commonly used in China, it is used to assess concurrent validity. The Pearson's correlation between TNS and NPTS was $0.766(P<0.001)$ and met the quality criteria, which indicated that the two scales showed good consistency. Due to the time, energy, and other objective constraints, convergent validity was not conducted. TNS scale can be used to assess the desired health outcomes, nurse-patient relationship, and patient satisfaction. ${ }^{9,17,38}$ Future studies should examine the correlations of Chinese version of TNS with other measures, such as European Quality of 5-Dimensions, Individualized Care Scale, and Inpatient Satisfaction Scale for Nursing to verify the validity of TNS.

The main limitation of this study was that it is confined to a single hospital, so selection bias may exist. Future studies should focus on a larger group of participants treated at different institutions. The lack of other measures for convergent validity evaluation, use of subsamples for test-retest analysis, and the short interval time between two surveys were additional limitations.

\section{Conclusion}

Our findings show that the Chinese version of the TNS can be used to measure the patients' trust in nurses in hospitalized patients with cancer. The TNS-4 had good internal consistency and structural validity, fair test-retest reliability, and good concurrent validity in a clinical sample of inpatients with cancer. The items of the scale are not disease specific and should therefore be applicable to all patients, and more research is needed to examine whether the scale is sufficiently sensitive in samples of patients with noncancer disease.

\section{Disclosure}

The authors report no conflicts of interest in this work.

\section{References}

1. Stewart BW, Wild CP. World Cancer Report 2014. Geneva, Switzerland: IARC Press, International Agency for Research on Cancer; 2014.

2. Chen W, Zheng R, Baade PD, et al. Cancer statistics in China, 2015 CA Cancer J Clin. 2016;66(2):115-132. 
3. Charalambous A, Radwin L, Berg A, et al. An international study of hospitalized cancer patients' health status, nursing care quality, perceived individuality in care and trust in nurses: a path analysis. Int $J$ Nurs Stud. 2016;61:176-186.

4. Sellman D. Trusting patients, trusting nurses. Nurs Philos. 2007; 8(1):28-36.

5. Bell L, Duffy A. A concept analysis of nurse-patient trust. Br J Nurs. 2009;18(1):46-51.

6. Miner-Williams D. Connectedness in the nurse-patient relationship: a grounded theory study. Issues Ment Health Nurs. 2007;28(11): 1215-1234.

7. Raeve LD. Trust and trustworthiness in nurse-patient relationships. Nurs Philos. 2002;3(2):152-162.

8. Dinc L, Gastmans C. Trust in nurse-patient relationships: a literature review. Nurs Ethics. 2013;20(5):501-516.

9. Benkert R, Hollie B, Nordstrom CK, Wickson B, Bins-Emerick L. Trust, mistrust, racial identity and patient satisfaction in urban African American primary care patients of nurse practitioners. J Nurs Scholarsh. 2009;41(2):211-219.

10. Shepherd ML. Behind the scales: child and family health nurses taking care of women's emotional wellbeing. Contemp Nurse. 2011;37(2): $137-148$.

11. Thorne SE, Robinson CA. Reciprocal trust in health care relationships. J Adv Nurs. 1988;13(6):782-789.

12. Mok E, Chiu PC. Nurse-patient relationships in palliative care. $J A d v$ Nurs. 2004;48(5):475-483.

13. Belcher M, Jones LK. Graduate nurses experiences of developing trust in the nurse-patient relationship. Contemp Nurse. 2009;31(2): $142-152$.

14. Altuntas S, Baykal U. Relationship between nurses' organizational trust levels and their organizational citizenship behaviors. J Nurs Scholarsh. 2010;42(2):186-194.

15. Li L, Zhong $\mathrm{H}$. Influence of holistic nursing care on confident degree in surgical nurse-patient relationship. J Nurs Sci. 1998;13(6):326-327.

16. Zhe-mei H, Wan-mei H, Li-hong C, Jian-ni L, Qun-di M. Analysis of reliability and validity of a nurse-patient trust scale. J Nurs Admin. 2010;10(10):700-701.

17. Radwin LE, Washko M, Suchy KA, Tyman K. Development and Pilot Testing of Four Desired Health Outcomes Scales. Oncol Nurs Forum. 2005;32(1):92-96.

18. Radwin LE, Cabral HJ. Trust in Nurses Scale: construct validity and internal reliability evaluation. J Adv Nurs. 2010;66(3):683-689.

19. Stolt M, Charalambous A, Radwin L, et al. Measuring trust in nursesPsychometric properties of the Trust in Nurses Scale in four countries. Eur J Oncol Nurs. 2016;25:46-54.

20. Jackson DL. Revisiting sample size and number of parameter estimates: some support for the N:q hypothesis. Struct Equ Modeling. 2003; 10(1):128-141.
21. Sousa VD, Rojjanasrirat W. Translation, adaptation and validation of instruments or scales for use in cross-cultural health care research: a clear and user-friendly guideline. J Eval Clin Pract. 2011;17(2):268-274.

22. Wu M. Structural Equation Modeling-the Operation and Application of Amos. 1st ed. Chongqing: Chongqing University Press; 2009.

23. McDonald RP, Ho MH. Principles and practice in reporting structural equation analyses. Psychol Methods. 2002;7(1):64-82.

24. DeVellis RF. Scale Development: Theory and Applications (Applied Social Research Methods). Thousand Oaks, CA: SAGE Publications; 2011.

25. Bowling A. Research Methods in Health: Investigating Health and Health Services. New York: Open University Press; 2014.

26. Charter RA. Testing the equality of two or more split-half reliability coefficients. Psychol Rep. 2001;88(3 pt 1):844-846.

27. Wu M. SPSS Statistical Application and Practice. Beijing: Science Press; 2003.

28. Giavarina D. Understanding Bland Altman analysis. Biochem Med (Zagreb). 2015;25(2):141-151.

29. Cohen J. Statistical power analysis for the behavioural sciences. J Am Stat Assoc. 1988;84(363):19-74.

30. Terwee CB, Bot SD, de Boer MR, et al. Quality criteria were proposed for measurement properties of health status questionnaires. J Clin Epidemiol. 2007;60(1):34-42.

31. Child D. The Essentials of Factor Analysis. 3rd ed. London: Continuum International Publishing Group; 2006.

32. Liu J, Sharen GW, Li J, Wang G. Dominance of the doctor-patient trust in the new healthcare reform background: virtuous trust and systemic trust. Medicine \& Philosophy. 2011;32(11):30-32.

33. National Health and Family Planning Commission of China [webpage on the Internet]. 2015 Statistic Bulletin of Health and Family Planning Development in China. Available from: http://www.moh.gov.cn/ guihuaxxs/s10748/201607/da7575d64fa04670b5f375c87b6229b0. shtml. Accessed December 20, 2016.

34. Brennan N, Barnes R, Calnan M, Corrigan O, Dieppe P, Entwistle V. Trust in the health-care provider-patient relationship: a systematic mapping review of the evidence base. Int J Qual Health Care. 2013; 25(6):682-688.

35. Cronbach LJ. Coefficient alpha and the internal structure of tests. Psychometrika. 1951;16(3):297-334.

36. Eisinga R, Grotenhuis M, Pelzer B. The reliability of a two-item scale: Pearson, Cronbach, or Spearman-Brown? Int J Public Health. 2013; 58(4):637-642.

37. Jones \& Bartlett Learning. Factor Analysis, Path Analysis, and Structural Equation Modeling. Available from: http://www.jblearning. com/samples/0763755486/55485_CH14_Walker.pdf. Accessed December 15, 2016

38. Ozaras G, Abaan S. Investigation of the trust status of the nurse-patient relationship. Nurs Ethics. Epub 2016 Sep 7:1-12.
Patient Preference and Adherence

\section{Publish your work in this journal}

Patient Preference and Adherence is an international, peer-reviewed, open access journal that focuses on the growing importance of patient preference and adherence throughout the therapeutic continuum. Patient satisfaction, acceptability, quality of life, compliance, persistence and their role in developing new therapeutic modalities and compounds to optimize

\section{Dovepress}

clinical outcomes for existing disease states are major areas of interest for the journal. This journal has been accepted for indexing on PubMed Central The manuscript management system is completely online and includes a very quick and fair peer-review system, which is all easy to use. Visit http://www. dovepress.com/testimonials.php to read real quotes from published authors. 